\title{
VISUAL DISTURBANCE OF THE UNCOVERED EYE IN PATIENTS WEARING AN EYE PATCH
}

\author{
R. B. ELLINGHAM, A. WALDOCK and R. A. HARRAD \\ Bristol
}

\begin{abstract}
SUMMARY
We questioned 104 consecutive eye casualty patients about any visual disturbance experienced while wearing an eye patch as part of their treatment. Many $(31 \%)$ reported momentary loss of vision in the uncovered eye. Some experienced this while driving. It was more common $(p<0.0008)$ if the 'dominant eye' had been patched $(47 \%)$ rather than the 'non-dominant eye' $(16 \%)$. In a separate experiment, a number of co-operative subjects were provided with a translucent-coloured occluder to be worn over each eye in turn. They reported a recurring coloured interference in the uncovered eye sparing the monocular crescent. We propose that the loss of vision in the unpatched eye is due to binocular rivalry suppression by the patched eye. This visual disturbance may be a cause of anxiety or risk for the patient. Patched patients should be warned this may occur, and advised to avoid driving or operating machinery.
\end{abstract}

It is well known that patients dislike wearing eye patches. This is particularly the case in young children being patched to treat strabismic amblyopia. It has been our experience, with adults treated with patches for abrasions in casualty, that certain patients describe transient losses of vision in the eye that is not wearing the eye patch. Although this phenomenon has been reported in the scientific literature it has received little comment, and has not previously been studied systematically or in a clinical context. Initial descriptions of this visual disturbance suggested interplay between the patched and unpatched eye.

The first few patients we questioned described three symptoms: transient blanking out of the vision, an intermittent sensation of fuzziness of the vision, like poor television reception ('snowstorm'), and intermittent blurring. All were similar to descriptions of binocular rivalry effects. Binocular rivalry is characterised by alternating periods of dominance and suppression when the two eyes receive incongruous information. The physiological locus for binocular rivalry is thought to be either in the visual cortex or the lateral geniculate body. ${ }^{1}$ There are, however,

Correspondence to: Richard A. Harrad, Bristol Eye Hospital, Lower Maudlin Street, Bristol BS1 2LX, UK. descriptions in the scientific literature of blanking out of vision when one eye is in darkness and the other views a uniform coloured field of illumination known as a Ganzfeld. Bolanowski and Doty ${ }^{2}$ described the loss of vision occurring when viewing an achromatic Ganzfeld with one eye while the other eye views a coloured Ganzfeld, as a periodic, transient drawing of a perceptual curtain nasotemporally across the visual field but sparing the far temporal crescent. Descriptions of retinal noise effects are often likened to a snow storm effect, or poor reception on a TV set. ${ }^{3}$ Retinal noise may be intrusive when, after a period of dark adaptation of the patched eye, it engages in binocular rivalry with the unpatched eye. Loss of visual sensation may also occur due to the fading of a stabilised retinal image with time. This is known as Troxler's effect, ${ }^{4}$ and is the reason why we are not constantly aware of the presence of our own retinal vasculature.

In order to gain a better understanding of the nature of the visual loss suffered by our patients, we designed a study to address the following questions:

1. Is there a specific phenomenon common to a large number of patients?

2. Does this phenomenon lead to significant symptoms or disability?

3. Can the phenomenon be ascribed to binocular rivalry suppression and is it related to eye dominance?

\section{METHODS}

Patients attending the Casualty Department at the Bristol Eye Hospital who had been instructed to wear an eye patch as part of their treatment were asked at review a series of questions about symptoms they may or may not have experienced during the time of patching. Patients were unselected with respect to symptoms, the eye condition indicating patching or which eye was patched. Consecutive patients seen by two of the doctors or two of the senior nurses were enrolled in the study. Inclusion criteria required that the eye patch was worn for at least 12 hours prior to review, but did not require that the patch had kept the covered eye closed. Not all patched patients could be 
included as a number were unable or unwilling to return for review.

Patients were not informed at the time of patching of the questions they would be asked later. They were thus not subject to suggestion. Questioners were masked to the patient's ocular dominance at the time of eliciting symptoms from the patient. Assessment of dominance was made at the end of questioning. In addition, as there is evidence to suggest that stereoacuity and binocular rivalry may be related, ${ }^{5}$ we assessed stereoacuity using the TNO chart.

At review the following details were documented for each patient on a questionnaire: name, age, eye condition and which eye was patched. Best corrected Snellen acuity for each eye was recorded. Patients were then directly questioned about any transient visual disturbances that may have occurred in the eye that was not patched. In particular they were asked about 'blanking out of vision', 'snowstorm' and 'blurring of vision'. These questions were designed to pick up specifically those symptoms corresponding to Ganzfeld-type blankout, retinal noise and binocular rivalry. If any of these had been experienced the patient was asked about their time of onset, duration, frequency and about any particular tasks that they made difficult. A personal and family history of amblyopia or strabismus was obtained. Finally ocular dominance was assessed by two methods. The first 60 patients in our series were asked which eye they preferred to use for one-eyed tasks. The last 44 patients were asked the same question, and carried out a simple test of ocular dominance using a cardboard tube, which is a modification of a standard technique. ${ }^{6}$ The patient lifts quickly, at arms length, the tube held in both hands. One eye of the examiner is thus quickly fixated by the patient, with both eyes open looking through the tube. It is clear which eye the subject chooses for fixation. The results of the test are highly consistent for individual patients. The results of the fixation test for the last 44 patients correlated perfectly with the patient's subjective ocular dominance.

Patients were initially assigned to two groups: (1) those with symptoms of visual disturbance and (2) those without such symptoms. These groups were further divided into (1) patients who had their dominant eye patched and (2) those who had their non-dominant eye patched. The numbers in the four subgroups were placed in a $2 \times 2$ table and compared using the chi-squared test.

In a separate experiment six observers in whom it was possible to determine eye dominance were asked to wear either a red- or green-coloured occluder over one eye. The translucent occluder was used to produce a Ganzfeld-type illumination. Each eye was tested in turn for a period of 20 minutes with a 10 minute rest interval. The occluders are constructed from half a table tennis ball shaped to fit snugly around the eye. They can be held in place with transparent colourless adhesive tape. The subjects were subsequently asked to describe any visual phenomena they may have experienced during the times of translucent occlusion.
In addition to this group of patients we were able to assess 8 adult amblyopes and 5 patients who had undergone enucleation of one eye around the time of our study. The enucleated patients were not patched but directly questioned in the same way as the padded casualty patients. The adult amblyopes had each eye patched in turn for a period of 30 minutes with 10 minutes rest in between. During the period of patching they were left watching television or reading a book in a dimly lit room.

\section{RESULTS}

Patches were affixed to patients during treatment for corneal abrasions (85\%), recurrent corneal erosions $(2 \%)$, following removal of ferrous particles $(8 \%)$ and minor chemical burns $(5 \%)$. One patient was patched to prevent diplopia of sudden onset. A third (31\%) of all patients described symptoms of visual disturbance, and in effect became repeatedly, transiently blind as a result of wearing the patch. One patient with blankout stated that this had been a particular problem when driving his car on the motorway. (It is our policy at Bristol Eye Hospital to warn patients not to drive when wearing an eye patch.) Most other patients with symptoms found them most troublesome when concentrating on reading or watching TV in dim ambient light. The numbers of each type of elicited symptom are shown in Table I and include both blankout and a sensation akin to viewing a television with intermittent poor reception (retinal noise). Most patients, however, described their symptom as transient blurring. Questioners were careful to ask whether patients thought that blurring was due to factors such as sympathetic watering of the uncovered eye or just blurring due to loss of binocular acuity. If this was the case then they were not included as having had the transient symptoms of visual disturbance due to the eye patch. Most patients with symptoms stated that the first onset of symptoms was soon (within minutes) after patching. A few were initially symptom-free for several hours.

The right eye was deemed to be dominant for 81 patients while 17 patients were deemed to be left eye dominant. For 6 of the 104 patients ocular dominance could not be determined using the tests described above. Three of these 6 patients had symptoms; the other 3 did not. All 6 were excluded from subsequent analysis. Fortyseven per cent (22/47) of patients with their dominant eye patched had symptoms of visual disturbance. Sixteen per cent $(8 / 51)$ of patients with their non-dominant eye patched also had symptoms, although none of these complained of blankout or TV type symptoms. Symptoms

Table I. Numbers of patched eye casualty patients reporting each of the transient symptoms consistent with perceptual blankout $(n=104)$

\begin{tabular}{lc}
\hline Symptom elicited on questioning & Number \\
\hline Blurring & 20 \\
Blankouts & 7 \\
'Snowstorm' & 2 \\
Combination of two symptoms & 4 \\
No symptoms & 71 \\
\hline
\end{tabular}


were significantly more prevalent in patients in whom the dominant eye was patched compared with those whose non-dominant eye was patched $(p<0.0008)$.

Analysing the data for the blankout and 'snowstorm' only (i.e. regarding those patients who described blurring as having experienced no symptoms) also shows a significant difference $(p<0.0025)$. This suggests that the presence of statistical significance is not an artefact produced by pooling all symptom types.

Visual acuities in the injured eyes were close to normal by the time of review. Although 7 of the 104 patients at review had Snellen acuities in the injured eye worse than $6 / 12$, while the fellow eye had a normal acuity, we felt this most likely to be due to the injury itself rather than undiagnosed amblyopia. Three of these had symptoms and 4 did not. Eight other patients in the group with symptoms had stereopsis worse than 480 seconds of arc when tested with the TNO stereo test. The percentages of patients with and without symptoms, grouped according to measured TNO stereoacuity performance, are illustrated in Fig. 1. The trend for the group with symptoms mirrors that for the group without symptoms. The presence of stereoacuity, pooled for all degrees of stereopsis, is no more frequent $(p<0.49)$ in the group with symptoms $(73 \%)$ than in the group without symptoms (79\%). None of the patients with symptoms gave a personal history of squint or amblyopia.

All subjects wearing coloured translucent occluders described interference in the uncovered eye which consisted of transient obscuration of the viewed scene by a curtain the colour of the translucent occluder, moving in a naso-temporal direction, sparing the far temporal visual field and sometimes the central vision. The latency of symptoms was shorter and frequency of symptoms greater when the dominant eye was covered for each subject. The respective values for latency ranged from 30 seconds to 4 minutes for a dominant eye, and from 3 minutes to $10 \mathrm{~min}$ utes for a non-dominant eye.

None of our amblyopic or enucleated patients described any symptom of visual disturbance.

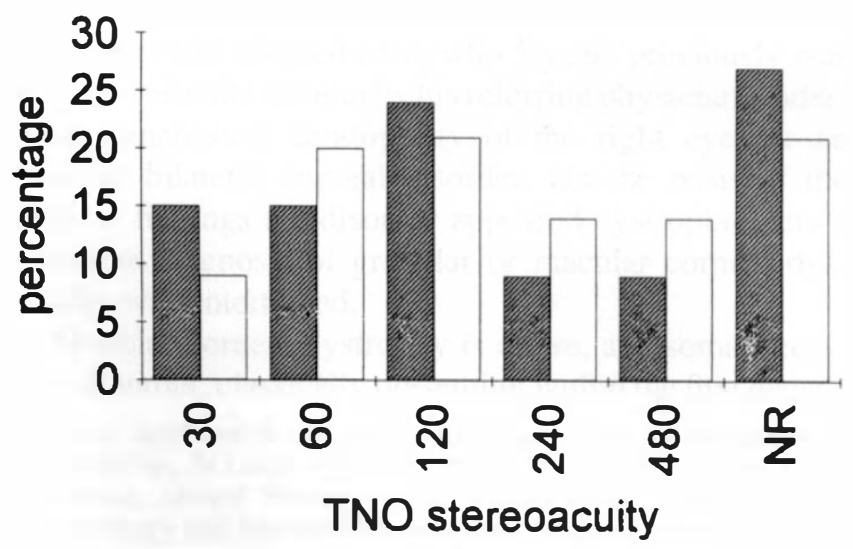

Fig. 1. Comparison for each TNO stereoacuity band of patients with symptoms (black bars) and patients without symptoms (open bars), in terms of the percentage of the total number of patients with and without symptoms, respectively. NR, not recordable.

\section{DISCUSSION}

Two possible explanations for the interference in the vision in the unpatched eye are binocular rivalry suppression of the uncovered eye, or a Troxler type fading of the image in the uncovered eye. The evidence for binocular rivalry suppression and against Troxler's effect is summarised as follows:

1. The reported visual disturbance was similar to that in the experiment when a coloured occluder was used to generate binocular rivalry and blankout of the uncovered eye. The effect produced had a similar periodicity and spared the monocular crescent.

2. When a subject who experienced some visual disturbance closed the unpatched eye it was still possible for him or her to see the pattern of the visual disturbance. This disturbance must, therefore, arise from the patched eye rather than be a fading of the image in the unpatched eye.

3 . Eye dominance is known to influence binocular rivalry ${ }^{2,6,7}$ and therefore the fact that the visual disturbance was more common in the patients who had the patch over the dominant eye was to be expected, if binocular rivalry suppression was operating. (The method we used for testing ocular dominance does not allow a subject to be classified as having mixed dominance and this may explain the finding that $8 / 30$ patients with their non-dominant eye patched had symptoms.)

4. The lack of any visual disturbance reported by patients who had recently undergone an enucleation $(n=5)$ is evidence against the visual disturbance being some sort of Troxler's effect.

5. On questioning the 8 amblyopic subjects we were surprised that they did not report binocular disturbance of their amblyopic eye when their normal eye was patched. We expected them to be strongly dominant in their normal eye and thus have obtrusive symptoms. The actual finding, however, is consistent with the theory that binocular rivalry is the underlying phenomenon, since these amblyopic subjects lacked binocular function and would not be expected to demonstrate binocular rivalry. ${ }^{8}$ We are currently studying a larger number of patients in order to ascertain the presence or absence of this phenomenon across a range of amblyopic subjects.

There is a debate in the psychophysical literature as to whether homogeneous fields can be strictly said to engage in binocular rivalry. ${ }^{2}$ What we have observed is an effect which consists of an interocular inhibitory process that shares many of the characteristics of binocular rivalry. However, in addition to creating disparate images in the patched and unpatched eye, an eye patch would also create two other special situations. Firstly, a well-patched eye will soon become dark adapted and an unpatched eye may not. Lansford and Baker ${ }^{9}$ reported in 1969 that light adaptation of one eye will under particular conditions lower visual stimulus thresholds during dark adaptation of the other eye. Makous et al. ${ }^{3}$ confirmed this finding, and using pressure blinding techniques (application of pressure to the globe until the optic nerve no longer conducts neural 
impulses - a reversible state) explained the results in terms of binocular rivalry. These authors did not find a reduction in threshold in all their subjects, and in the light of our findings this could perhaps be explained in terms of ocular dominance. They concluded that a uniocularly darkadapted eye sends signals to the brain that interfere with the detection of signals received by the other eye. They distinguished this from the ordinary discrimination of signal from noise at a retinal level. Secondly, a well-patched eye should be closed. Twenty-four per cent of our patients with symptoms stated that their eye pad did not keep the eye closed underneath, as opposed to $8 \%$ of patients without symptoms. This difference achieves significance $(p<0.028)$. The roles of dark adaptation and eye closure in the patched eye situation remain to be explained.

\section{CONCLUSION}

The phenomena described here may help to explain why patients dislike wearing eye patches. We feel that visual disturbance of the uncovered eye of patients wearing an eye patch is a real phenomenon, due to binocular rivalry, which may be a cause of distress for the patient. It may also be relevant in other clinical situations where one eye is covered, as in uniocular visual field testing, and in day case surgery where a patient may go home with a patch over one eye. In particular, patients receiving eye patches as part of their treatment in Casualty Departments should be warned of the possible effects of the patch, and advised not to drive or operate machinery.
The authors wish to thank Patricia Ferguson for providing the initial stimulus to the study, the Bristol Eye Hospital casualty nurses for assisting with questionnaires and Alistair Laidlaw for helpful comments on the manuscript.

Key words: Binocular rivalry, Blankout, Ganzfeld, Ocular dominance, Patching, Suppression, Troxler's effect.

\section{REFERENCES}

1. Fox R. Binocular rivalry. In: Regan D, editor. Vision and visual dysfunction, Vol. 9. London: Macmillan Press 1991: 93-110.

2. Bolanowski SJ, Doty RW. Perceptual 'blankout' of monocular homogeneous fields (Ganzfelder) is prevented with binocular viewing. Vision Res 1987;27:967-82.

3. Makous W, Teller D, Boothe R. Binocular interaction in the dark. Vision Res 1976;16:473-6.

4. Troxler D. Über das Verschwinden gegebener Gegenstände innerhalb unsers Gesichtskreises. Ophthal Bibliothek 1804;2: 51-3.

5. Halpern DL, Patterson R, Blake R. Are stereoacuity and binocular rivalry related? Am J Optom Physiol Opt 1987;64: $41-4$.

6. Ogle KN. Ocular dominance and binocular retinal rivalry. In: Davson H, editor. Davson's physiology of the eye. 2nd ed. London: Macmillan Press; 1963;409-17.

7. Levelt WJM. On binocular rivalry. Institute for Perception, unpublished doctoral thesis, University of Utrecht, RVOTNO Soesterberg, The Netherlands, 1965.

8. Mein J, Trimble R. Diagnosis and management of ocular motility disorders. 2nd ed. Oxford: Blackwell Scientific, 1991.

9. Lansford TG, Baker HD. Dark adaptation: an interocular light-adaptation effect. Science 1969;164:1307-9. 